\title{
A Jarník-type theorem for a problem of approximation by cubic polynomials
}

by

$$
\text { Alessandro Pezzoni (York) }
$$

1. Introduction. Classical Diophantine approximation investigates the density of the rational numbers in the set of real numbers, starting with Dirichlet's theorem, which states that for any real number $x$ there are infinitely many pairs $(p, q) \in \mathbb{Z} \times \mathbb{Z} \backslash\{0\}$ such that

$$
|q x-p|<1 / q \text {. }
$$

This is in a sense optimal, since by Hurwitz's theorem,

$$
|q \varphi-p|<\frac{1}{c q}
$$

has at most finitely many solutions $(p, q)$ as above when $c>\sqrt{5}$ and $\varphi=$ $(\sqrt{5}-1) / 2$ (see [6, Theorem 194] for a proof). However, Khinchin's and Jarník's theorems tell us - in a very precise way - how likely it is that a randomly chosen real number can be approximated by rationals up to a certain accuracy, which is given in terms of a decreasing function of the denominators.

More precisely, let $\psi: \mathbb{R}^{+} \rightarrow \mathbb{R}^{+}$be such a function, called an approximation function, and define

$$
W_{n}(\psi):=\left\{x \in \mathbb{R}^{n}:|q \cdot x-p|<\psi(|q|) \text { for i.m. }(p, q) \in \mathbb{Z} \times \mathbb{Z}^{n} \backslash\{0\}\right\}
$$

where $|q|=\max \left|q_{i}\right|$ and "i.m." is shorthand for "infinitely many". Here and in what follows, $\mathcal{H}^{s}$ denotes the usual $s$-dimensional Hausdorff measure, which we recall in the next section.

2020 Mathematics Subject Classification: Primary 11J83.

Key words and phrases: Diophantine approximation, Hausdorff measure, Mahler's problem, cubic polynomials.

Received 26 September 2018; revised 8 April 2019.

Published online 29 January 2020.

The work is licensed under a Creative Commons Attribution (CC-BY) license. 
TheOrem 1.1 (Jarník). In the above setting, for any $s \geq 0$ we have

$$
\mathcal{H}^{s}\left(W_{1}(\psi)\right)= \begin{cases}0 & \text { if } \sum_{q=1}^{\infty} \psi(q)^{s} q^{1-s}<\infty, \\ \infty & \text { otherwise. }\end{cases}
$$

Furthermore, with some extra hypotheses (detailed in Corollary 1.3 below) Jarník's theorem can be generalised to Hausdorff $g$-measures $\mathcal{H}^{g}$, and this gives a pretty accurate description of the geometry of the set $W_{1}(\psi)$. A more general approximation problem consists in looking at the set $\mathscr{L}_{n}(w)$ of real numbers $x$ such that

$$
|P(x)|<\mathrm{H}(P)^{-w}
$$

for infinitely many integer polynomials $P$ with degree bounded above by $n$, where $w>0$ is given and $\mathrm{H}(P)$ denotes the height of $P$, i.e. the maximum absolute value of its coefficients. This is related to, but subtly different from, the problem of approximating $x$ by algebraic numbers of bounded degree; we refer the interested reader to [4, Chapter 3, in particular the last part of Section 3.4].

The study of this problem dates back to Mahler. With Minkowski's linear forms theorem one can prove that $\mathscr{L}_{n}(w)$ has full Lebesgue measure for any $w \leq n$, and in 1932 Mahler [12] conjectured that $\mathscr{L}_{n}(w)$ has measure 0 for every $w>n$. In 1969 Sprindžuk [14] proved this in full generality, although the cases $n=2$ and $n=3$ had already been settled by Kubilius, Kasch, and Volkmann (see [15] for more details).

The picture for Hausdorff measures, on the other hand, is a bit less clear. In 1983 Bernik [3] proved that $\mathscr{L}_{n}(w)$ has Hausdorff dimension $\frac{n+1}{w+1}$, and in 2006 Beresnevich, Dickinson, and Velani proved [2, Theorem 18], which specialises to the divergence part of a Jarník-type theorem for Mahler's problem. Interestingly, though, the convergence case is not quite as straightforward as for Jarník's theorem and the only results so far in this direction are for $n=2$; these were the work of Hussain [8] and Huang [7], who gave a more general proof for the case of non-degenerate $\mathcal{C}^{2}$ plane curves.

1.1. Hausdorff measures and dimension. Let $X$ be a subset of $\mathbb{R}^{n}$ and let $g: \mathbb{R}^{+} \rightarrow \mathbb{R}^{+}$be a dimension function, i.e. a continuous, increasing function such that $g(r) \rightarrow 0$ as $r \rightarrow 0$. Given $\rho>0$, a $\rho$-cover of $X$ is a (possibly countable) collection $\left\{B_{i}\right\}$ of balls of $\mathbb{R}^{n}$ such that the radius $r\left(B_{i}\right)$ of each ball $B_{i}$ lies in $(0, \rho]$ and $X \subseteq \bigcup B_{i}$. Now define

$$
\mathcal{H}_{\rho}^{g}(X):=\inf \left\{\sum g\left(r\left(B_{i}\right)\right):\left\{B_{i}\right\} \text { is a } \rho \text {-cover of } X\right\}
$$

and note that this is increasing as $\rho \rightarrow 0$. Therefore the limit

$$
\mathcal{H}^{g}(X):=\lim _{\rho \rightarrow 0^{+}} \mathcal{H}_{\rho}^{g}(X)=\sup _{\rho>0} \mathcal{H}_{\rho}^{g}(X)
$$


exists, and is called the Hausdorff $g$-measure of $X$. When $g(r)=r^{s}$ for some $s \geq 0$, it is customary to write $\mathcal{H}^{s}(X)$ for $\mathcal{H}^{g}(X)$, which is then called the $s$-dimensional Hausdorff measure of $X$. Moreover, if $s$ is an integer, then $\mathcal{H}^{s}$ is just a constant multiple of the Lebesgue measure on $\mathbb{R}^{s}$.

If $h, g$ are two dimension functions, a straightforward standard argument shows that if $h(r) / g(r) \rightarrow 0$ when $r \rightarrow 0$, then

$$
\mathcal{H}^{h}(X)=0 \quad \text { whenever } \quad \mathcal{H}^{g}(X)<\infty .
$$

In particular, this implies that if $s>t \geq 0$ and $\mathcal{H}^{t}(X)<\infty$, then $\mathcal{H}^{s}(X)=0$. We can then define the Hausdorff dimension of $X$ as

$$
\operatorname{dim}_{H}(X):=\inf \left\{s \geq 0: \mathcal{H}^{s}(X)=0\right\}=\sup \left\{s \geq 0: \mathcal{H}^{s}(X)=\infty\right\} .
$$

Finally, note that if $\operatorname{dim}_{\mathrm{H}}$ is an integer, then it coincides with the usual "naive" notion of dimension.

1.2. Our setting. From here on we will make heavy use of Vinogradov's notation $\ll$ and $\asymp$, where $a \ll b$ means that $a \leq c b$ for some constant $c>0$, while $a \asymp b$ means that both $a \ll b$ and $b \ll a$. We will also use subscripts to emphasise the dependence of the implied constant on certain quantities; for example, $a \ll_{n} b$ means that the implied constant $c$ depends on $n$. For later convenience, define

$$
\begin{aligned}
\mathcal{P}_{n} & :=\{P \in \mathbb{Z}[X]: \operatorname{deg}(P) \leq n\}, \\
\mathcal{A}_{n}(\psi) & :=\left\{x \in \mathbb{R}:|P(x)| \leq \psi(\mathrm{H}(P)) \text { for i.m. } P \in \mathcal{P}_{n}\right\} .
\end{aligned}
$$

Theorem 1.2 (Beresnevich, Dickinson, Velani [2, Theorem 18]). Let $\mathcal{M}$ be a non-degenerate submanifold of $\mathbb{R}^{n}$ of dimension $m$. Let $\psi$ be an approximation function, and let $g$ be a dimension function such that $q^{-m} g(q)$ is decreasing and $q^{-m} g(q) \rightarrow \infty$ as $q \rightarrow 0$. Furthermore, suppose that $q^{1-m} g(q)$ is increasing. Then

$$
\mathcal{H}^{g}\left(W_{n}(\psi) \cap \mathcal{M}\right)=\infty \quad \text { if } \quad \sum_{q=1}^{\infty} g\left(\frac{\psi(q)}{q}\right) \psi(q)^{1-m} q^{m+n-1}=\infty .
$$

COROLlaRY 1.3. Let $\psi$ be an approximation function and consider an increasing dimension function $g$ such that $q^{-1} g(q)$ is decreasing and $q^{-1} g(q)$ $\rightarrow \infty$ as $q \rightarrow 0$. Then

$$
\mathcal{H}^{g}\left(\mathcal{A}_{n}(\psi)\right)=\infty \quad \text { if } \quad \sum_{q=1}^{\infty} g\left(\frac{\psi(q)}{q}\right) q^{n}=\infty .
$$

Recall that the discriminant of an integer polynomial $P$ of degree $n$ is defined as

$$
\mathrm{D}(P):=a_{n}^{2 n-2} \prod_{1 \leq i<j \leq n}\left(\alpha_{i}-\alpha_{j}\right)^{2}
$$


where $\alpha_{1}, \ldots, \alpha_{n}$ are the (possibly complex) roots of $P$, counted with multiplicity, and $a_{n}$ is the leading coefficient of $P$. Furthermore, it can be shown that $\mathrm{D}(P)$ is a homogeneous polynomial of degree $2 n-2$ in the coefficients of $P$, and its value is bounded above by $c_{n} \mathrm{H}(P)^{2 n-2}$ for some constant $c_{n}$ that depends only on $n$.

Now, for any given $0<\lambda \leq n-1$ and fixed $0<\tau \leq c_{n}$, consider

$$
\begin{aligned}
\mathcal{P}_{n}^{\lambda} & :=\left\{P \in \mathcal{P}_{n}:|\mathrm{D}(P)| \geq \tau \mathrm{H}(P)^{2(n-1-\lambda)}\right\}, \\
\mathcal{A}_{n}^{\lambda}(\psi) & :=\left\{x \in \mathbb{R}:|P(x)| \leq \psi(\mathrm{H}(P)) \text { for i.m. } P \in \mathcal{P}_{n}^{\lambda}\right\} .
\end{aligned}
$$

In this paper we will examine the case $n=3$ of the convergence equivalent of Corollary 1.3 and provide a partial result for general $n$; moreover, our conclusions do not depend on the choice of $\tau$. Namely, we will prove the following:

Theorem 1.4. Let $\psi$ and $g$ be as in Corollary 1.3 . Then for any $0<$ $\lambda<1$ we have

$$
\mathcal{H}^{g}\left(\mathcal{A}_{n}^{\lambda}(\psi)\right)=0 \quad \text { if } \quad \sum_{q=1}^{\infty} g\left(\frac{\psi(q)}{q}\right) q^{n}<\infty .
$$

For the counterpart, set

$$
\begin{aligned}
\mathcal{P}_{n, \lambda} & :=\left\{P \in \mathcal{P}_{n}:|\mathrm{D}(P)|<\tau \mathrm{H}(P)^{2(n-1-\lambda)}\right\}, \\
\mathcal{A}_{n, \lambda}(\psi) & :=\left\{x \in \mathbb{R}:|P(x)| \leq \psi(\mathrm{H}(P)) \text { for i.m. } P \in \mathcal{P}_{n, \lambda}\right\} .
\end{aligned}
$$

Theorem 1.5. Consider $\psi$ and $g$ as in Corollary 1.3. Let $\mathcal{P}_{3, \lambda}^{*}$ be the set of irreducible polynomials in $\mathcal{P}_{3, \lambda}$ and let $\mathcal{A}_{3, \lambda}^{*}(\psi)$ be the corresponding limsup set. Further assume that $0 \leq \lambda<9 / 20$. Then

$$
\mathcal{H}^{g}\left(\mathcal{A}_{3, \lambda}^{*}(\psi)\right)=0 \quad \text { if } \quad \sum_{q=1}^{\infty} g\left(\frac{\psi(q)}{q}\right) q^{3-2 \lambda / 3}<\infty .
$$

COROLlary 1.6. Suppose that $\psi(q)=q^{-w}$ for some $w>0$ and that $0 \leq \lambda<9 / 20$. As is customary, write $\mathcal{A}_{3, \lambda}(w)$ for $\mathcal{A}_{3, \lambda}(\psi)$. Then

$$
\mathcal{H}^{g}\left(\mathcal{A}_{3, \lambda}(w)\right)=0 \quad \text { if } \quad \sum_{q=1}^{\infty} g\left(q^{-w-1}\right) q^{3-2 \lambda / 3}<\infty .
$$

Note that the condition $0 \leq \lambda<9 / 20$ stems from the fact that our proof is based on the discriminant estimate from [10, Corollary 2], much like Volkmann's proof of the cubic case of Mahler's conjecture [15] relied on a similar estimate by Davenport [5]. As a special case, for $\lambda=0$ we recover Bernik's result for $n=3$, namely that the Hausdorff dimension of $\mathscr{L}_{3}(w)=\mathcal{A}_{3,0}(w)$ is $\frac{4}{w+1}$. 
2. A few lemmas on polynomials. In this section we will collect some lemmas that we will use later in this paper. Some we prove here, while others are taken from [14, often restated in a slightly simpler way that is enough for our purpose.

Lemma 2.1 ([16, Hilfssatz 3]). Let $P_{1}, \ldots, P_{k}$ be integer polynomials. Then

$$
\mathrm{H}\left(P_{1} \cdots P_{k}\right) \asymp \mathrm{H}\left(P_{1}\right) \cdots \mathrm{H}\left(P_{k}\right)
$$

where the implied constants depend only on the degrees of the polynomials.

Proof. Recall that the Mahler measure of a polynomial $P$ of degree $d$ is defined as

$$
\mathrm{M}(P):=\left|a_{d}\right| \prod_{i=1}^{d} \max \left\{1,\left|\alpha_{i}\right|\right\}
$$

where $a_{d}$ and $\alpha_{i}$ are the leading coefficient and roots of $P$, respectively. Now, Mahler [13] showed that

$$
\left(\begin{array}{c}
d \\
\lfloor d / 2\rfloor
\end{array}\right)^{-1} \mathrm{H}(P) \leq \mathrm{M}(P) \leq \sqrt{d+1} \mathrm{H}(P) .
$$

Hence the result follows by noting that the Mahler measure is multiplicative, which can be easily seen from its definition.

Lemma 2.2 ([11, Lemma 3]). Let $P$ be an integer polynomial of degree at most $n \geq 2$ and with non-zero discriminant. If $\alpha$ is a root of $P$, then

$$
\left|P^{\prime}(\alpha)\right| \gg|\mathrm{D}(P)|^{1 / 2} \mathrm{H}(P)^{-n+2}
$$

where the implied constant depends only on $n$.

Lemma 2.3 ([11, Lemma 4]). Let $P$ be as in Lemma 2.2 and let $x \in \mathbb{C}$. If $\alpha$ is the root of $P$ closest to $x$, then

$$
|x-\alpha| \ll \mathrm{H}(P)^{n-2}|\mathrm{D}(P)|^{-1 / 2}|P(x)|
$$

where the implied constant depends only on $n$.

Lemma 2.4. In the setting of Lemma 2.3 write $H$ for $\mathrm{H}(P)$. Furthermore, assume that $x \in[-1 / 2,1 / 2)$ and $|P(x)|<\psi(H)$ for some approximation function $\psi$. If $|\mathrm{D}(P)| \gg_{n} H^{2(n-1-\lambda)}$ for some $0<2 \lambda<1-\log _{H} \psi(H)$, then $\left|P^{\prime}(x)\right| \asymp_{n}\left|P^{\prime}(\alpha)\right|$ for sufficiently large $H$.

Proof. First, observe that by Lemma 2.2 we have

$$
\left|P^{\prime}(\alpha)\right| \gg_{n} H^{n-1-\lambda} H^{-n+2}=H^{1-\lambda} .
$$

Then note that by Lemma 2.3 .

$$
|x-\alpha| \ll_{n} H^{n-2} H^{\lambda+1-n} \psi(H)=H^{\lambda-1} \psi(H)<H^{-1 / 2} \psi(H)^{1 / 2} .
$$


Hence we can assume $|\alpha|<1$, since this is less than $1 / 2$ for $H$ large enough. Now, by the mean value theorem we can find some $z$ between $x$ and $\alpha$, thus with $|z|<1$, such that

$$
\left|P^{\prime}(x)-P^{\prime}(\alpha)\right|=\left|P^{\prime \prime}(z)\right||x-\alpha| \ll_{n} H H^{\lambda-1} \psi(H)=H^{\lambda} \psi(H) .
$$

Finally, the hypothesis on $\lambda$ implies that $H^{\lambda} \psi(H)<H^{1-\lambda}$, therefore up to choosing $H$ large enough we have

$$
\left|P^{\prime}(x)-P^{\prime}(\alpha)\right|<\frac{1}{2}\left|P^{\prime}(\alpha)\right|,
$$

from which it follows that $\left|P^{\prime}(x)\right| \asymp_{n}\left|P^{\prime}(\alpha)\right|$, as required.

Lemma 2.5. Fix $P \in \mathbb{C}[X]$ and $m \in \mathbb{C}$. If $P(X)=a_{n} X^{n}+\cdots+a_{1} X+a_{0}$, then the coefficients of $P(X+m)=b_{n} X^{n}+\cdots+b_{n} X+b_{0}$ are

$$
b_{k}=\sum_{j=k}^{n}\left(\begin{array}{l}
j \\
k
\end{array}\right) a_{j} m^{j-k} \quad \text { for each } 0 \leq k \leq n .
$$

Proof. We proceed by induction on $n$. If $n=1$ then $P(X+m)=a_{1} X+$ $a_{0}+m a_{1}$, which agrees with the above formula. Now assume the lemma is true for $n-1$. Since we can write $P(X)=a_{n} X^{n}+Q(X)$, where $Q(X)=$ $a_{n-1} X^{n-1}+\cdots+a_{0}$, we have

$$
P(X+m)=Q(X+m)+a_{n}(X+m)^{n}=Q(X+m)+a_{n} \sum_{i=0}^{n}\left(\begin{array}{c}
n \\
i
\end{array}\right) X^{i} m^{n-i} .
$$

Thus $b_{n}=a_{n}$ and, by the induction hypothesis, for $0 \leq k \leq n-1$,

$$
b_{k}=\left(\begin{array}{l}
n \\
k
\end{array}\right) a_{n} m^{n-k}+\sum_{j=k}^{n-1}\left(\begin{array}{l}
j \\
k
\end{array}\right) a_{j} m^{j-k}=\sum_{j=k}^{n}\left(\begin{array}{l}
j \\
k
\end{array}\right) a_{j} m^{j-k} .
$$

Note. While we chose to state the lemma over $\mathbb{C}$ for simplicity, there is nothing specific to it in the proof, which carries over as-is for any other commutative ring with unity.

COROLlary 2.6. In the setting of Lemma 2.5 we have

$$
\mathrm{H}(P(X+m)) \leq(1+|m|)^{n} \mathrm{H}(P) .
$$

Proof. By Lemma 2.5, for each $0 \leq k \leq n$ we have

$$
\left|b_{k}\right| \leq \sum_{j=k}^{n}\left(\begin{array}{l}
j \\
k
\end{array}\right)\left|a_{j}\right||m|^{j-k} \leq \mathrm{H}(P) \sum_{j=k}^{n}\left(\begin{array}{l}
j \\
k
\end{array}\right)|m|^{j-k} .
$$

Since $(1+|m|)^{n}=\sum_{s=0}^{n}\left(\begin{array}{c}n \\ s\end{array}\right)|m|^{s}$, it is enough to prove that

$$
\left(\begin{array}{l}
n \\
s
\end{array}\right) \geq\left(\begin{array}{c}
s+k \\
k
\end{array}\right)=\left(\begin{array}{c}
s+k \\
s
\end{array}\right)
$$


for any $0 \leq k \leq n$ and $0 \leq s \leq n-k$. On the other hand, $\left(\begin{array}{l}t \\ s\end{array}\right)$ is monotonic in $t$ for $t \geq s$, which can be readily seen from

$$
\left(\begin{array}{c}
t+1 \\
s
\end{array}\right)=\frac{t+1}{t+1-s} \frac{t !}{(t-s) ! s !} \geq\left(\begin{array}{l}
t \\
s
\end{array}\right) .
$$

Therefore the observation that $n \geq j=s+k$ completes the proof.

3. Proof of Theorem 1.4. Let $I:=[-1 / 2,1 / 2)$. We prove the result for $\mathcal{A}_{n}^{\lambda}(\psi) \cap I$, and then extend it to the whole $\mathcal{A}_{n}^{\lambda}(\psi)$. Our first goal is to estimate how much each polynomial in $\mathcal{P}_{n}^{\lambda}$ can contribute towards $\mathcal{A}_{n}^{\lambda}(\psi)$. To do so, consider some $\varepsilon>0$ and $Q \in \mathbb{N}$. For a polynomial $P \in \mathcal{P}_{n}^{\lambda}$ with $\mathrm{H}(P) \leq Q$ define

$$
\sigma_{\varepsilon}(P):=\left\{x \in I:|P(x)| \leq \varepsilon,\left|P^{\prime}(x)\right| \geq 2\right\} .
$$

Then let $B_{n}(Q, \varepsilon)$ be the union of $\sigma_{\varepsilon}(P)$ over all such polynomials. We will rely on the following specialisation of [1, Proposition 1]:

Lemma 3.1. For any $Q>4 n^{2}$ and any $\varepsilon<n^{-1} 2^{-n-2} Q^{-n}$ we have

$$
\left|B_{n}(Q, \varepsilon)\right| \leq n 2^{n+2} \varepsilon Q^{n},
$$

where $\left|B_{n}(Q, \varepsilon)\right|$ denotes the Lebesgue measure of $B_{n}(Q, \varepsilon)$.

Now, partition $\mathcal{P}_{n}^{\lambda}$ into sets

$$
\mathcal{P}_{n}^{\lambda}(t):=\left\{P \in \mathcal{P}_{n}^{\lambda}: 2^{t} \leq \mathrm{H}(P)<2^{t+1}\right\}
$$

and observe that

$$
\mathcal{A}_{n}^{\lambda}(\psi) \cap I=\limsup \gamma_{\psi}(P)=\bigcap_{t_{0}=1}^{\infty} \bigcup_{t=t_{0}}^{\infty} \bigcup_{P \in \mathcal{P}_{n}^{\lambda}(t)} \gamma_{\psi}(P)
$$

where $\gamma_{\psi}(P):=\{x \in I:|P(x)| \leq \psi(\mathrm{H}(P))\}$. Then, for $t$ large enough and for any $P \in \mathcal{P}_{n}^{\lambda}(t)$, letting $\varepsilon=\psi\left(2^{t}\right)$ we have $\gamma_{\psi}(P) \subseteq \sigma_{\varepsilon}(P)$, so that the sets $\sigma_{\varepsilon}(P)$ form a cover of $\mathcal{A}_{n}^{\lambda}(\psi) \cap I$. Indeed, $\psi(\mathrm{H}(P)) \leq \varepsilon$ since $\psi$ is assumed to be decreasing. Furthermore, if $\alpha$ is the root of $P$ closest to $x$, then up to choosing $t_{0}$ large enough Lemma 2.4 ensures that $\left|P^{\prime}(x)\right|$ is comparable to $\left|P^{\prime}(\alpha)\right|$, hence

$$
\left|P^{\prime}(x)\right| \gg_{n} \mathrm{H}(P)^{1-\lambda} \geq 2^{t(1-\lambda)}
$$

so $\left|P^{\prime}(x)\right|>2$, again up to choosing $t_{0}$ large enough.

Note that each $\sigma_{\varepsilon}(P)$ is a union of finitely many intervals, the number of which is bounded above by a constant that depends only on $n$. We cannot use this directly to obtain an upper bound for the Hausdorff dimension of $\mathcal{A}_{n}^{\lambda}$, though, because those intervals can be arbitrarily small, and also we do not know how many polynomials there are in each $\mathcal{P}_{n}^{\lambda}(t)$. To fix this, consider 
the sets

$$
\tilde{\sigma}_{\varepsilon}(P):=\bigcup_{x \in \sigma_{\varepsilon}(P)}\left\{y \in I:|y-x|<2^{-t} \varepsilon\right\} .
$$

Clearly $\sigma_{\varepsilon}(P) \subseteq \tilde{\sigma}_{\varepsilon}(P)$. Furthermore, by the mean value theorem, for each $y \in \tilde{\sigma}_{\varepsilon}(P)$ there is a $z \in I$ which lies between $y$ and the corresponding $x \in \sigma_{\varepsilon}(P)$ such that

$$
|P(y)-P(x)|=\left|P^{\prime}(z)\right||y-x| .
$$

Since $|z|<1$, we have $\left|P^{\prime}(z)\right| \ll_{n} \mathrm{H}(P)<2^{t+1}$, thus

$$
|P(y)| \leq|P(x)|+\left|P^{\prime}(z)\right||y-x| \ll_{n}|P(x)|+2 \varepsilon \ll \varepsilon .
$$

Now, let $c$ be the constant implied in the above inequality, so that $\sigma_{\varepsilon}(P)$ is covered by intervals in $\sigma_{c \varepsilon}(P)$ of length at least $\ell=2^{1-t} \varepsilon$. From this we can obtain a cover made up of intervals of length exactly $\ell$, splitting up the larger intervals and allowing some overlap at the edges as necessary, and by Lemma 3.1 the polynomials in $\mathcal{P}_{n}^{\lambda}(t)$ contribute at most

$$
\frac{\left|B_{n}\left(2^{t+1}, c \varepsilon\right)\right|}{\ell} \ll_{n} 2^{t(n+1)}=: N
$$

of these intervals. To conclude, it follows that

$$
\begin{aligned}
\mathcal{H}^{g}\left(\mathcal{A}_{n}^{\lambda} \cap I\right) & \ll_{n} \lim _{t_{0} \rightarrow \infty} \sum_{t \geq t_{0}} g(\ell) N=\lim _{t_{0} \rightarrow \infty} \sum_{t \geq t_{0}} g\left(\frac{\psi\left(2^{t}\right)}{2^{t-1}}\right) 2^{t(n+1)} \\
& \leq \lim _{t_{0} \rightarrow \infty} \sum_{t \geq t_{0}} g\left(\frac{\psi\left(2^{t}\right)}{2^{t}}\right) 2^{t(n+1)}=0
\end{aligned}
$$

because $g$ is assumed to be increasing, $\psi$ is decreasing, and by Cauchy's condensation test we know that

$$
\sum_{t \geq 0} g\left(\frac{\psi\left(2^{t}\right)}{2^{t}}\right) 2^{t(n+1)}<\infty \quad \text { iff } \quad \sum_{q \geq 1} g\left(\frac{\psi(q)}{q}\right) q^{n}<\infty .
$$

3.1. Extending the argument. Fix $m \in \mathbb{Z}$ and consider $x \in[m-1 / 2$, $m+1 / 2)$. Then suppose that $P \in \mathcal{P}_{n}^{\lambda}$ is such that $|P(x)| \leq \psi(\mathrm{H}(P))$. Now, note that $y=x-m \in I$ and let $Q(X)=P(X+m)$, so that $Q(y)=P(x)$. Furthermore, by Corollary 2.6 we know that $c \mathrm{H}(Q) \leq \mathrm{H}(P)$, where $c=$ $(1+|m|)^{-n}$ is independent of $P$. Therefore $Q \in \mathcal{P}_{n}^{\lambda}$ and

$$
|Q(y)| \leq \psi(\mathrm{H}(P)) \leq \psi(c \mathrm{H}(Q)) .
$$

Hence the following lemma, together with the previous argument, is enough to complete the proof of Theorem 1.4 . 
Lemma 3.2. Let $0<c_{1}<c_{2}$. Then

$$
\sum_{q=1}^{\infty} g\left(\frac{\psi\left(c_{1} q\right)}{q}\right) q^{n}<\infty \quad \text { iff } \quad \sum_{q=1}^{\infty} g\left(\frac{\psi\left(c_{2} q\right)}{q}\right) q^{n}<\infty .
$$

Proof. To begin, assume that the series with $c_{1}$ converges. Since $\psi$ is decreasing we have $\psi\left(c_{1} q\right) \geq \psi\left(c_{2} q\right)$, and since $g$ is increasing it follows that

$$
\sum_{q=1}^{\infty} g\left(\frac{\psi\left(c_{2} q\right)}{q}\right) q^{n} \leq \sum_{q=1}^{\infty} g\left(\frac{\psi\left(c_{1} q\right)}{q}\right) q^{n}<\infty .
$$

For the other implication, note that $c=c_{2} c_{1}^{-1}>1$ and

$$
\begin{aligned}
\sum_{q \geq c} g\left(\frac{\psi\left(c_{1} q\right)}{q}\right) q^{n} & =\sum_{r=1}^{\infty} \sum_{c r \leq q<c(r+1)} g\left(\frac{\psi\left(c_{1} q\right)}{q}\right) q^{n} \\
& \leq \sum_{r=1}^{\infty} \sum_{c r \leq q<c(r+1)} g\left(\frac{\psi\left(c c_{1} r\right)}{c r}\right) c^{n}(r+1)^{n} \\
& \leq 2^{n} c^{n} \sum_{r=1}^{\infty}\left(\sum_{c r \leq q<c(r+1)} 1\right) g\left(\frac{\psi\left(c_{2} r\right)}{r}\right) r^{n} \\
& \leq 2^{n} c^{n+1} \sum_{r=1}^{\infty} g\left(\frac{\psi\left(c_{2} r\right)}{r}\right) r^{n}
\end{aligned}
$$

where the first two inequalities are again due to the fact that $g$ is increasing and $\psi$ is decreasing. Therefore the first series converges when the second does, as required.

4. Proof of Theorem 1.5. Just as in the proof of Theorem 1.4, we will focus on $\mathcal{A}_{n, \lambda}^{*}(\psi) \cap I$, after which the result immediately extends to the whole $\mathcal{A}_{n, \lambda}^{*}(\psi)$. Similarly to what we did there, define

$$
\mathcal{P}_{3, \lambda}^{*}(t):=\left\{P \in \mathcal{P}_{3, \lambda}^{*}: 2^{t} \leq \mathrm{H}(P)<2^{t+1}\right\} .
$$

Now suppose that $P \in \mathcal{P}_{3, \lambda}^{*}$ and let $\sigma(P)$ be the set of $x \in I$ such that $|P(x)| \leq \psi(\mathrm{H}(P))$. Furthermore, let $\sigma(t)$ be the union of $\sigma(P)$ over all $P$ in $\mathcal{P}_{3, \lambda}^{*}(t)$. Then, by Lemma 2.3 , we know that

$$
|x-\alpha| \leq c \mathrm{H}(P)|\mathrm{D}(P)|^{-1 / 2} \psi(\mathrm{H}(P))=: r(P, \psi)
$$

where $\alpha$ is the root of $P$ closest to $x$ and where the constant $c>0$ is independent of $P$ and $x$. Hence $\sigma(P)$ is covered by at most three intervals 
of radius $r(P, \psi)$ centred at the roots of $P$. Then

$$
\mathcal{A}_{3, \lambda}^{*}(\psi) \cap I \subseteq \limsup \sigma(t)=\bigcap_{t_{0}=0}^{\infty} \bigcup_{t=t_{0}}^{\infty} \sigma(t)
$$

and

$$
\begin{aligned}
|\sigma(t)| & \leq \sum_{P \in \mathcal{P}_{3, \lambda}^{*}(t)}|\sigma(P)| \ll \sum_{P \in \mathcal{P}_{3, \lambda}^{*}(t)} \mathrm{H}(P)|\mathrm{D}(P)|^{-1 / 2} \psi(\mathrm{H}(P)) \\
& \ll 2^{t} \psi\left(2^{t}\right) \sum_{P \in \mathcal{P}_{3, \lambda}^{*}(t)}|\mathrm{D}(P)|^{-1 / 2} \ll 2^{t(3-2 \lambda / 3)} \psi\left(2^{t}\right)
\end{aligned}
$$

because from [10, Corollary 2] it follows immediately that

$$
\sum_{P \in \mathcal{P}_{3, \lambda}^{*}(t)}|\mathrm{D}(P)|^{-1 / 2} \asymp 2^{t(2-2 \lambda / 3)}
$$

where the implied constants are absolute.

Just as in the proof of Theorem 1.4, consider a slight enlargement of $\sigma(P)$ :

$$
\tilde{\sigma}(P)=\bigcup_{x \in \sigma(P)}\left\{y \in \mathbb{R}:|y-x|<\psi\left(2^{t}\right) / 2^{t}\right\},
$$

so that for any $y \in \tilde{\sigma}(P)$ we have

$$
|P(y)| \leq|P(x)|+\left|P^{\prime}(z)\right||x-y| \ll \psi(\mathrm{H}(P)) .
$$

Thus $\sigma(P) \subseteq \tilde{\sigma}(P)$ and $|\tilde{\sigma}(t)| \asymp|\sigma(t)|$. It follows that we can cover $\sigma(t)$ with at most

$$
N:=\frac{|\tilde{\sigma}(t)|}{\ell} \ll 2^{t(3-2 \lambda / 3)} \psi\left(2^{t}\right) \frac{2^{t}}{\psi\left(2^{t}\right)}=2^{t(4-2 \lambda / 3)}
$$

intervals of length $\ell:=\psi\left(2^{t}\right) / 2^{t}$. Finally, this implies that

$$
\mathcal{H}^{g}\left(\mathcal{A}_{3, \lambda}(\psi) \cap I\right) \ll \lim _{t_{0} \rightarrow \infty} \sum_{t=t_{0}}^{\infty} g\left(\frac{\psi\left(2^{t}\right)}{2^{t}}\right) 2^{t(4-2 \lambda / 3)}=0
$$

since by Cauchy's condensation test we know that

$$
\sum_{t=0}^{\infty} g\left(\frac{\psi\left(2^{t}\right)}{2^{t}}\right) 2^{t(4-2 \lambda / 3)}<\infty \quad \text { iff } \sum_{q=1}^{\infty} g\left(\frac{\psi(q)}{q}\right) q^{3-2 \lambda / 3}<\infty .
$$

5. Proof of Corollary 1.6. By Theorem 1.5 it is enough to focus on reducible polynomials, i.e. on $\mathcal{B}:=\mathcal{A}_{3, \lambda}(w) \backslash \mathcal{A}_{3, \lambda}^{*}(w)$. Now consider $x \in \mathbb{R}$ such that $|P(x)| \leq \mathrm{H}(P)^{-w}$ for infinitely many reducible cubic polynomials $P$ and write $P=P_{1} P_{2}$ with $\operatorname{deg}\left(P_{i}\right)=i$. Note that if, say, $\left|P_{1}(x)\right| \leq \mathrm{H}\left(P_{1}\right)^{-w}$ for at most finitely many $P_{1}$, then $\left|P_{1}(x)\right| \gg \mathrm{H}\left(P_{1}\right)^{-w}$ for all $P_{1}$ and by Lemma 2.1 
we have

$$
\mathrm{H}\left(P_{1}\right)^{-w}\left|P_{2}(x)\right| \ll\left|P_{1}(x) P_{2}(x)\right| \leq \mathrm{H}(P)^{-w} \ll \mathrm{H}\left(P_{1}\right)^{-w} \mathrm{H}\left(P_{2}\right)^{-w} .
$$

It follows that for at least one $i \in\{1,2\}$ we can find a constant $c_{i}>0$ such that $\left|P_{i}(x)\right| \leq c_{i} \mathrm{H}\left(P_{i}\right)^{-w}$ for infinitely many $P_{i}$. In other words,

$$
\mathcal{B} \subseteq \mathcal{A}_{1}\left(c_{1} q^{-w}\right) \cup \mathcal{A}_{2}\left(c_{2} q^{-w}\right) .
$$

Similarly, since a quadratic polynomial is either irreducible or a product or two linear polynomials, we can also find constants $c_{i}^{\prime}>0$ such that

$$
\mathcal{A}_{2}\left(c_{2} q^{-w}\right) \subseteq \mathcal{A}_{1}\left(c_{1}^{\prime} q^{-w}\right) \cup \mathcal{A}_{2}^{*}\left(c_{2}^{\prime} q^{-w}\right)
$$

where $\mathcal{A}_{2}^{*}=\mathcal{A}_{2,0}^{*}$. Furthermore, without loss of generality we may assume that $c_{1} \geq c_{1}^{\prime}$, so that

$$
\mathcal{B} \subseteq \mathcal{A}_{1}\left(c_{1} q^{-w}\right) \cup \mathcal{A}_{2}^{*}\left(c_{2}^{\prime} q^{-w}\right) .
$$

Then Jarník's theorem implies that

$$
\mathcal{H}^{g}\left(\mathcal{A}_{1}\left(c_{1} q^{-w}\right)\right)=0 \text { if } \sum_{q=1}^{\infty} g\left(c_{1} q^{-w-1}\right) q<\infty,
$$

and the proof of $[8$, Case II] implies that

$$
\mathcal{H}^{g}\left(\mathcal{A}_{2}^{*}\left(c_{2}^{\prime} q^{-w}\right)\right)=0 \text { if } \sum_{q=1}^{\infty} g\left(c_{2}^{\prime} q^{-w-1}\right) q^{2}<\infty .
$$

Finally, by the comparison test and by Lemma 3.2, those two series converge when

$$
\sum_{q=1}^{\infty} g\left(q^{-w-1}\right) q^{2}<\infty
$$

This is enough to complete the proof of Corollary 1.6 since $0 \leq \lambda<9 / 20$ means that $3-2 \lambda / 3>2$.

6. Conclusions. The main issue with proving a convergence result in the case of reducible polynomials for more general approximation functions, similar to what we did for Corollary 1.6. lies in decoupling the resulting inequalities

$$
\left|P_{1}(x)\right|\left|P_{2}(x)\right| \leq \psi\left(\mathrm{H}\left(P_{1} P_{2}\right)\right) .
$$

Our proof carries through as-is for any other $\psi$ that is multiplicative, but this is by no means the general case. For the case of quadratic polynomials, Hussain [8] and Huang [7] resorted to imposing a fairly restrictive condition on the dimension function, which looks artificial; however, in private correspondence Hussain confirmed that the techniques used in those papers do not allow for its removal. Furthermore, in a recent preprint Hussain, Schleischitz and Simmons [9] showed that this decoupling can be achieved in the 
general case when, for all $q$ large enough, $\log \psi(q) / \log q$ is non-increasing and for every $c_{1}>1$ there is a $c_{2}>0$ such that $\psi\left(q / c_{1}\right) \leq c_{2} \psi(q)$. Obviously these are satisfied for $\psi(q)=q^{-w}$ and multiplicative functions satisfy the latter condition, but it is not immediately clear whether multiplicative approximation functions need to satisfy the former condition.

It would also be interesting to look into an equivalent version of Theorem 1.5 for higher degrees, which would lead to a complete treatment of the case of approximation functions of the form $q^{-w}$. However, an analogue of the discriminant estimate from [10] appears necessary to apply our method in higher degrees, hence this will likely require some different techniques, just like Sprindžuk's solution of Mahler's conjecture required techniques different from those Volkmann used in his treatment of the cubic case.

Acknowledgements. The author is supported by EPSRC International Doctoral Scholars IDS Grant EP/N509802/1.

\section{References}

[1] V. Beresnevich, On approximation of real numbers by real algebraic numbers, Acta Arith. 90 (1999), 97-112.

[2] V. Beresnevich, D. Dickinson and S. Velani, Measure theoretic laws for lim sup sets, Mem. Amer. Math. Soc. 179 (2006), no. 846, x+91 pp.

[3] V. I. Bernik, Application of the Hausdorff dimension in the theory of Diophantine approximations, Acta Arith. 42 (1983), 219-253 (in Russian).

[4] Y. Bugeaud, Approximation by Algebraic Numbers, Cambridge Tracts in Math. 160, Cambridge Univ. Press, Cambridge, 2004.

[5] H. Davenport, A note on binary cubic forms, Mathematika 8 (1961), 58-62.

[6] G. H. Hardy and E. M. Wright, An Introduction to the Theory of Numbers, 6th ed., Oxford Univ. Press, Oxford, 2008.

[7] J.-J, Huang, Hausdorff theory of dual approximation on planar curves, J. Reine Angew. Math. 740 (2018), 63-76.

[8] M. Hussain, A Jarnik type theorem for planar curves: everything about the parabola, Math. Proc. Cambridge Philos. Soc. 159 (2015), 47-60.

[9] M. Hussain, J. Schleischitz and D. Simmons, Diophantine approximation on curves, arXiv:1902.02094v1 (2019).

[10] D. Kaliada, F. Götze and O. Kukso, The asymptotic number of integral cubic polynomials with bounded heights and discriminants, Lithuanian Math. J. 54 (2014), 150165.

[11] F. Kasch und B. Volkmann, Zur Mahlerschen Vermutung über S-Zahlen, Math. Ann. 136 (1958), 442-453.

[12] K. Mahler, Über das Maß der Menge aller S-Zahlen, Math. Ann. 106 (1932), 131-139.

[13] K. Mahler, On two extremum properties of polynomials, Illinois J. Math. 7 (1963), 681-701.

[14] V. G. Sprindžuk, Mahler's Problem in Metric Number Theory, Transl. Math. Monogr. 25, Amer. Math. Soc., Providence, RI, 1969.

[15] B. Volkmann, The real cubic case of Mahler's conjecture, Mathematika 8 (1961), $55-57$. 
[16] E. Wirsing, Approximation mit algebraischen Zahlen beschränkten Grades, J. Reine Angew. Math. 206 (1961), 67-77.

\author{
Alessandro Pezzoni \\ Department of Mathematics \\ University of York \\ York, YO10 5DD, United Kingdom \\ E-mail: alessandro.pezzoni@runbox.com
}


